\title{
Optical implementation of the two-dimensional fractional Fourier transform with different orders in the two dimensions
}

\author{
Aysegul Sahin ${ }^{a}$, Haldun M. Ozaktas ${ }^{\text {a }}$, David Mendlovic ${ }^{b}$ \\ a Department of Electrical Engineering. Bilkent University. TR-06533 Bilkent. Ankara. Turkey \\ b Faculty of Engineering, Tel-Aviv University, 69978 Tel-Aviv, Israel
}

Received 26 April 1995

\begin{abstract}
Previous optical implementations of the two-dimensional fractional Fourier transform have assumed identical transform orders in both dimensions. We let the orders in the two orthogonal dimensions to be different and present general design formulae for optically implementing such transforms. This design formulae allows us to specify the two orders and the input, output scale parameters simultaneously.
\end{abstract}

\section{Introduction}

The fractional Fourier transform of order $a$ is defined in a manner such that the common Fourier transform is a special case with order $a=1$ [1]. The one-dimensional fractional Fourier transform of order $a$ can be defined for $0<|a|<2$ as

$$
\begin{aligned}
& \mathcal{F}^{a}[\{f(u)\}](u)=\int_{-\infty}^{\infty} B_{a}\left(u, u^{\prime}\right) f\left(u^{\prime}\right) \mathrm{d} u^{\prime}, \\
& B_{a}\left(u, u^{\prime}\right)=\frac{\exp [-\mathrm{j}(\pi \hat{\phi} / 4-\phi / 2)]}{\sqrt{|\sin \phi|}} \exp \left[\mathrm{j} \pi\left(u^{2} \cot \phi-2 u u^{\prime} \csc \phi+u^{\prime 2} \cot \phi\right)\right],
\end{aligned}
$$

where $\phi=a \pi / 2$ and $\hat{\phi}=\operatorname{sgn}(\sin \phi)$. The kernel is defined separetely for $a=0$ and $a=2$ as $B_{0}\left(u, u^{\prime}\right)=$ $\delta\left(u-u^{\prime}\right)$ and $B_{2}\left(u, u^{\prime}\right)=\delta\left(u+u^{\prime}\right)$. The definition can easily be extended outside the interval $[-2,2]$ by noting that $\mathcal{F}^{4 j+a}(u)=\mathcal{F}^{a}(u)$.

Some essential properties of the fractional Fourier transform are: (i) It is linear. (ii) The first order transform ( $a=1$ ) corresponds to the common Fourier transform. (iii) It is additive in index, $\mathcal{F}^{a_{1}} \mathcal{F}^{a_{2}} \hat{q}=\mathcal{F}^{a_{1}+a_{2}} \hat{q}$.

The fractional Fourier transforming property of quadratic graded index media was discussed in Refs. [1$5,17]$. Bulk optical systems that realize fractional Fourier transforms were presented in Refs. $[7,8,10]$. Several applications of fractional Fourier transforms were presented in Refs. [1,6,7,13]. It was also shown that the 
fractional Fourier transform can be used to describe optical beam propagation and as a tool for analyzing optical systems composed of thin lenses and sections of free space [9,14-16].

The definition of the 2-D fractional Fourier transform is generally made by using the same order in both directions. In this paper we define the 2-D fractional Fourier transform with different orders in the two dimensions. The kernel for this transform is nothing but the product of two 1-D kernels. The 2-D fractional Fourier transform with order $a_{x}$ along the $x$ axis and $a_{y}$ in the $y$ axis is defined as

$$
F^{a_{x}, a_{v}}[\{f(u, v)\}](u, v)=\int_{-\infty}^{\infty} \int_{-\infty}^{\infty} B_{a_{x}, a_{y}}\left(u, v ; u^{\prime}, v^{\prime}\right) f\left(u^{\prime}, v^{\prime}\right) \mathrm{d} u^{\prime} \mathrm{d} v^{\prime}
$$

where

$$
B_{a_{x}, a_{y}}\left(u, v ; u^{\prime}, v^{\prime}\right)=B_{a_{x}}\left(u, u^{\prime}\right) B_{a_{y}}\left(v, v^{\prime}\right) .
$$

We interpret $u, u^{\prime}, v, v^{\prime}$ as dimensionless variables.

Such anamorphic fractional Fourier transforms are necessary in order to generalize previously suggested optical signal processing and filtering schemes to two dimensions [1-5,13]. Since the characteristics of the signals in the two dimensions may vary, the optimal filtering may require fractional transforms with different orders in the two dimensions. Schemes for realizing anamorphic optical fractional Fourier transforms of variable orders are also essential for tomographic complex wave-field reconstruction techniques $[11,12]$.

In this study an optical system for performing the 2-D fractional Fourier transform with different orders is presented. This system allows us to realize 2-D fractional Fourier transform with the desired orders and input, output scale parameters.

\section{Optical system for performing 2-D fractional Fourier transform}

The suggested system which realizes two-dimensional fractional Fourier transform can be seen in Fig. 1. Each anamorphic lens appearing in the figure may be composed of two orthogonally situated cylindrical thin lenses with different focal lengths.

In the following analysis we determine the focal lengths and separations of the lenses so that $p_{\text {out }}(x, y)$ is the 2-D fractional Fourier transform of $p_{\text {in }}(x, y)$. Let $p_{1}(x, y)$ denote the light distribution after a propagation of distance $d_{1}$ in free space. $p_{\text {in }}(x, y)$ and $p_{1}(x, y)$ are related to each other by a simple convolution relation:

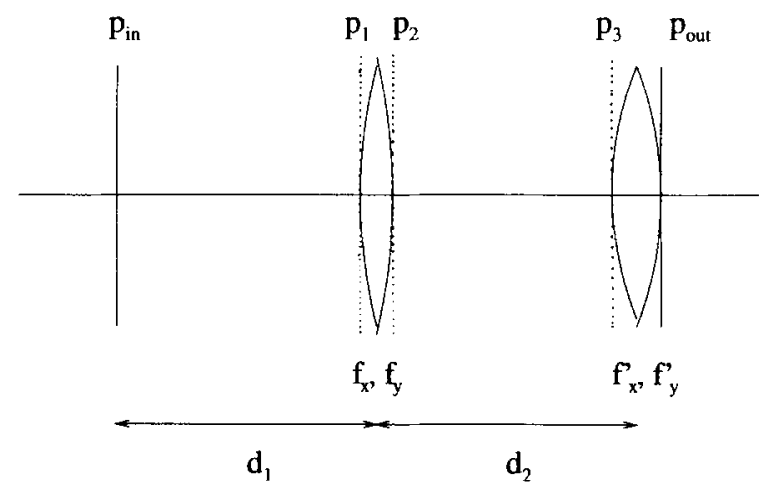

Fig. 1. The optical system 


$$
p_{1}(x, y)=\exp (-\mathrm{j} \pi / 2) \frac{1}{\lambda d} \exp (\mathrm{j} k d) \int_{-\infty}^{\infty} \int_{-\infty}^{\infty} p_{\text {in }}\left(x^{\prime}, y^{\prime}\right) \exp \left\{\mathrm{j} \pi\left[\left(x-x^{\prime}\right)^{2}+\left(y-y^{\prime}\right)^{2}\right] / \lambda\right\} \mathrm{d} x^{\prime} \mathrm{d} y^{\prime} .
$$

The first lens in the figure has focal length $f_{x}$ in the $x$ direction and $f_{y}$ in the $y$ direction. The light distribution immediate to the right of the first lens is:

$$
p_{2}(x, y)=p_{1}(x, y) \exp \left[-\mathrm{j} \frac{\pi}{\lambda}\left(\frac{x^{2}}{f_{x}}+\frac{y^{2}}{f_{y}}\right)\right] .
$$

Propagation in the second section of free space results in another convolution. The light distribution just before the second lens is:

$$
p_{3}(x, y)=\exp (-\mathrm{j} \pi / 2) \frac{1}{\lambda d} \exp (\mathrm{j} k d) \int_{-\infty}^{\infty} \int_{-\infty}^{\infty} p_{2}\left(x^{\prime \prime}, y^{\prime \prime}\right) \exp \left\{\mathrm{j} \pi\left[\left(x-x^{\prime \prime}\right)^{2}+\left(y-y^{\prime \prime}\right)^{2}\right] / \lambda\right\} \mathrm{d} x^{\prime \prime} \mathrm{d} y^{\prime \prime} .
$$

The second lens has focal lengths $f_{x}^{\prime}$ and $f_{y}^{\prime}$. This final lens will not affect the light intensity. If we are interested in only the light intensity, there is no need to use these lenses at the output.

After some algebraic manipulations $p_{\text {out }}(x, y)$ can be written in terms of $p_{\text {in }}(x, y)$ as follows:

$$
p_{\text {out }}(x, y)=\int_{-\infty}^{\infty} \int_{-\infty}^{\infty} K \exp \left[\mathrm{j} \pi\left(x^{2} A_{1}-2 x x^{\prime} A_{2}+x^{\prime 2} A_{3}+y^{2} B_{1}-2 y y^{\prime} B_{2}+y^{\prime 2} B_{3}\right)\right] p_{\text {in }}\left(x^{\prime}, y^{\prime}\right) \mathrm{d} x^{\prime} \mathrm{d} y^{\prime},
$$

where

$$
\begin{aligned}
& K=\frac{\exp (-\mathrm{j} \pi / 2) \exp \left[\mathrm{j} k\left(d_{1}+d_{2}\right)\right]}{\lambda} \sqrt{\frac{f_{x} f_{y}}{\left(d_{2} f_{x}+d_{1} f_{x}-d_{1} d_{2}\right)\left(d_{2} f_{y}+d_{1} f_{y}-d_{1} d_{2}\right)},} \\
& A_{1}=\frac{f_{x}-d_{1}}{\lambda\left(d_{2} f_{x}-d_{1} f_{x}-d_{1} d_{2}\right)}-\frac{1}{\lambda f_{x}^{\prime}}, \quad A_{2}=\frac{f_{x}}{\lambda\left(d_{2} f_{x}-d_{1} f_{x}-d_{1} d_{2}\right)}, \quad A_{3}=\frac{f_{x}-d_{2}}{\lambda\left(d_{2} f_{x}-d_{1} f_{x}-d_{1} d_{2}\right)}, \\
& B_{1}=\frac{f_{y}-d_{1}}{\lambda\left(d_{2} f_{y}-d_{1} f_{y}-d_{1} d_{2}\right)}-\frac{1}{\lambda f_{y}^{\prime}}, \quad B_{2}=\frac{f_{y}-d_{1}}{\lambda\left(d_{2} f_{y}-d_{1} f_{y}-d_{1} d_{2}\right)}, \quad B_{3}=\frac{f_{y}-d_{1}}{\lambda\left(d_{2} f_{y}-d_{1} f_{y}-d_{1} d_{2}\right)} .
\end{aligned}
$$

Remember that $x, x^{\prime}, y, y^{\prime}$ etc. have dimensions of length. Thus, in order to put this equation in a form comparable with Eq. (2), we need to introduce the dimensionless variables $u_{1}=x / s_{1}, u_{2}=x^{\prime} / s_{2}, v_{1}=y / s_{1}$ and $v_{2}=y^{\prime} / s_{2}$. Here $s_{1}$ and $s_{2}$ are real valued positive scale parameters with dimensions of length. Also introducing the hatted functions $\hat{p}_{\text {out }}\left(u_{1}, v_{1}\right)=p_{\text {out }}\left(u_{1} s_{1}, v_{1} s_{1}\right)$ and $\hat{p}_{\text {in }}\left(u_{2}, v_{2}\right)=p_{\text {in }}\left(u_{2} s_{2}, v_{2} s_{2}\right)$, Eq. (8) can be written as

$$
\begin{gathered}
\hat{p}_{\text {out }}\left(u_{1}, v_{1}\right)=\int_{-\infty}^{\infty} \int_{-\infty}^{\infty} s_{2}^{2} K \exp \left[\mathrm{j} \pi\left(u_{1}^{2} s_{1}^{2} A_{1}-2 u_{1} u_{2} s_{1} s_{2} A_{2}+u_{2}^{2} s_{2}^{2} A_{3}\right]\right. \\
\times \exp \left[\mathrm{j} \pi\left(v_{1}^{2} s_{1}^{2} B_{1}-2 v_{1} v_{2} s_{1} s_{2} B_{2}+v_{2}^{2} s_{2}^{2} B_{3}\right)\right] \hat{p}_{\text {in }}\left(u_{2}, v_{2}\right) \mathrm{d} u_{2} \mathrm{~d} v_{2} .
\end{gathered}
$$

We wish this to represent a fractional Fourier transform relation with the specified orders and scale parameters. By comparing Eq. (3) and Eq. (10), we obtain the following necessary and sufficient equations:

$$
\begin{array}{lll}
s_{1}^{2} A_{1}=\cot \phi_{x}, & s_{1} s_{2} A_{2}=\csc \phi_{x}, & s_{2}^{2} A_{3}=\cot \phi_{x}, \\
s_{1}^{2} B_{1}=\cot \phi_{y}, & s_{1} s_{2} B_{2}=\csc \phi_{y}, & s_{2}^{2} B_{3}=\cot \phi_{y} .
\end{array}
$$


These equations can be solved after some straightforward yet lengthy algebraic manipulations. If $\phi_{x}=\phi_{y}$, the equations will simplify and one recovers Lohmann's results [10]. When $\phi_{x} \neq \phi_{y}$, these equations can be solved for the lens focal lengths and separations as functions of the desired orders and scale parameters:

$$
\begin{aligned}
& d_{1}=\frac{s_{2}^{2}\left(\sin \phi_{y}-\sin \phi_{x}\right)}{\lambda\left(\cos \phi_{y}-\cos \phi_{x}\right)}, \\
& d_{2}=\frac{s_{1} s_{2} \sin \left(\phi_{x}-\phi_{y}\right)}{\lambda\left(\cos \phi_{y}-\cos \phi_{x}\right)}, \\
& f_{x}=\frac{s_{1} s_{2}^{2} \sin \left(\phi_{x}-\phi_{y}\right)}{\lambda\left(s_{2}-s_{1} \cos \phi_{x}\right)\left(\cos \phi_{y}-\cos \phi_{x}\right)}, \\
& f_{y}=\frac{s_{1} s_{2}^{2} \sin \left(\phi_{x}-\phi_{y}\right)}{\lambda\left(s_{2}-s_{1} \cos \phi_{y}\right)\left(\cos \phi_{y}-\cos \phi_{x}\right)}, \\
& f_{x}^{\prime}=\frac{s_{2} \sin \left(\phi_{x}-\phi_{y}\right)}{\lambda\left[s_{1}\left(\cos \phi_{y}-\cos \phi_{x}\right)+s_{2}\left(1-\cos \left(\phi_{y}-\phi_{x}\right)\right)\right]}, \\
& f_{y}^{\prime}=\frac{s_{2} \sin \left(\phi_{x}-\phi_{y}\right)}{\lambda\left[s_{1}\left(\cos \phi_{y}-\cos \phi_{x}\right)+s_{2}\left(\cos \left(\phi_{y}-\phi_{x}\right)-1\right)\right]} .
\end{aligned}
$$

Thus by choosing the focal lengths and separations of the lenses as given above, we can optically realize a two-dimensional fractional Fourier transform with orders $\phi_{x}, \phi_{y}$ and scale parameters $s_{1}$ and $s_{2}$. Notice that $d_{1}$ and $d_{2}$, as given by Eq. (12) and Eq. (13), may turn out to be negative. In such cases we would have to deal with virtual objects and/or images. This would require the use of additional lenses. In the event that we wish to avoid this, we must require that $d_{1}$ and $d_{2}$ be positive. This will then restrict the range of $a_{x}$ and $a_{y}$, that can be realized. This range can be maximized by allowing the $x$ and $y$ axes to be flipped. For instance, with $\phi_{x}=60$ and $\phi_{y}=30$, we obtain a negative $d_{1}$ value. This transform is equivalent to the fractional Fourier transform with $\phi_{x}=60$ and $\phi_{y}=210$ followed by a flip of the $y$ axis. (This is because a transform of order 2 corresponds to a flip of the coordinate axis.) In order to implement some orders, both axes should be flipped. Fig. 2 shows the necessary flip(s) required to realize different combinations of $\mathrm{n}^{-1}$.m

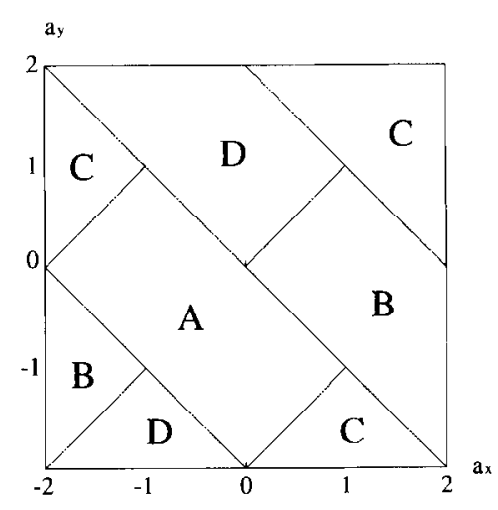

Fig. 2. A: no flip; B: one flip; C: two flips; D: inaccessible. 


\section{Conclusion}

The 2-D fractional Fourier transform is defined with different orders in the two dimensions. An optical system that realizes such 2-D fractional Fourier transforms is presented (Fig. 1). Our system is flexible enough to allow one to specify the orders in both dimensions and the input, output scale parameters independently. Most combinations of orders can be reached by using the suggested optical system. The final design equations are given in Fig. 1.

\section{References}

[1] H.M. Ozaktas, B. Barshan, D. Mendlovic and L. Onural, J. Opt. Soc. Am. A 11 (1994) 547.

[2] H.M. Ozaktas and A.W. Lohmann, Optical Computing, OSA 1993 Technical Digest Series Vol. 7 (Optical Society of America, Washington, D.C., 1993) p. 127.

[3] H.M. Ozaktas and D. Mendlovic, Optics Comm. 101 (1993) 163.

[4] D. Mendlovic and H.M. Ozaktas, J. Opt. Soc. Am. A 10 (1993) 1875.

[5] D. Mendlovic and H.M. Ozaktas, J. Opt. Soc. Am. A 10 (1993) 2522.

[6] A.W. Lohmann and B.H. Soffer, J. Opt. Soc. Am. A 11 (1994) 1798.

[7] L.M. Bernardo and O.D.D. Soares, Optics Comm. 110 (1994) 517.

[8] L.M. Bernardo and O.D.D. Soares, J. Opt. Soc. Am. A 11 (1994) 2622.

[9] H.M. Ozaktas and D. Mendlovic, J. Opt. Soc. Am. A 12 (1995) 743.

[10] A.W. Lohmann, J. Opt. Soc. Am. A 10 (1993) 2181.

[11] M.G. Raymer, M. Beck and D.F. McAlister, Phys. Rev. Lett. 72 (1994) 1137.

[12] D.T. Smithey, M. Beck, A. Faridani and M.G. Raymer, Phys. Rev. Lett. 70 (1993) 1244.

[13] M.A. Kutay, H.M. Ozaktas, O. Arikan and L. Onural, Optimal filtering in fractional Fourier domains, IEEE Transactions on Signal Processing, submitted.

[14] H.M. Ozaktas and D. Mendlovic, Optics Lett. 19 (1994) 1678.

[15] P. Pellat-Finet, Optics Lett. 19 (1994) 1388.

[16] P. Pellat-Finet and G. Bonnet, Optics Comm. 111 (1994) 141.

[17] M.F. Erden, H.M. Ozaktas and D. Mendlovic, J. Opt. Soc. Am. A., submitted. 